\title{
POEMS SYNDROME WITH TRANSIENT INTRACRANIAL HYPERTENSION AND NONSPECIFIC GAIT DISTURBANCE
}

\author{
Vivek Rauniyar, Chen Li and Huan Yang
}

\begin{abstract}
POEMS syndrome (polyneuropathy, organomegaly, endocrinopathy, monoclonal or M-protein and skin changes) is a rare multisystem disorder associated with plasma cell dyscrasia and have a wide range of clinical features and presentations. The clinical manifestations are so varied that many of them could be present at the time of diagnosis or only one could be present and followed by appearance of others making early correct diagnosis very difficult. Here, we describe a case of POEMS syndrome that first had skin changes and later developed most of the features along with transient highly raised intracranial pressure (ICP) along with gait disturbance which are unusual clinical findings and have rarely been reported in the literature.
\end{abstract}

Key words: POEMS Syndrome, Plasma cell dyscrasia, intracranial pressure (ICP).

\section{Introduction}

POEMS syndrome, also known as CrowFukase syndrome, is a rare multisystem paraneoplastic disorder secondary to an underlying plasma cell dyscrasia [1,2,]. The major presenting clinical features comprises of endocrinopathy and chronic progressive polyneuropathy with predominant motor disability [3]. POEMS acronym stands forpolyneuropathy, organomegaly, endocrinopathy, $\mathrm{M}$ protein, and skin changes, which are classical features of the disease [4]. Other important features include sclerotic bone lesions, castleman disease, papilledema, peripheral edema, ascites, pleural effusions, thrombocytosis, polycythemia, fatigue and clubbing [4].
The pathogenesis of this multisystem disease is complex, but recently some progress has been made in understanding of its pathophysiological basis. Elevations of proangiogenic and pro-inflammatory cytokines are the hallmark of this disorder [5, 6]. POEMS syndrome is thought to be a consequence of the secretion and resultant action of the pro-inflammatory cytokines such as interleukin (IL)-1ם, IL-6, tumor necrosis factor (TNF) and vascular endothelial growth factor(VEGF) which are often detected in excess amounts in patients and are thought to be responsible for many of the clinical features of the disease[4,5,6].

Here, we report a case of POEMS syndrome with unusually transiently raised intracranial 
pressure along with nonspecific gait disturbance.

\section{Case Report}

A 35-year-old woman presented to us with 6 weeks history of progressive gait difficulties associated with bilateral distal paresthesia in her lower limbs that gradually made her difficult to walk. Physical examination revealed areflexia in all four-limbs along with mild symmetrical motor deficits (muscle power MRC grade 4 in both lower limbs and 5 in both upper limbs) and decreased muscle tone with intact superficial and deep sensation. No Pathological reflexes were noted.

The patient had significant history of patchy hyperpigmentation of skin especially of the distal upper limbs for one year and was diagnosed hypothyroidism 4 weeks back. At this presentation detailed workup showed she had hepato-splenomegaly, minimal bilateral pleural effusion, minimal pericardial effusion and ascites. She had bilateral pitting edema of the lower limbs and also complained of prolonged irregular menstrual cycle. Moreover, she also had significant history of weight loss. Bilateral papilledema was also noted. She had no complaints of headache. No meningeal signs were present during examination. Her brain magnetic resonance(MR) image(T2 coronal image at the level of lateral ventricles) showed the ventricles were normal in shape and size(figure 1) .

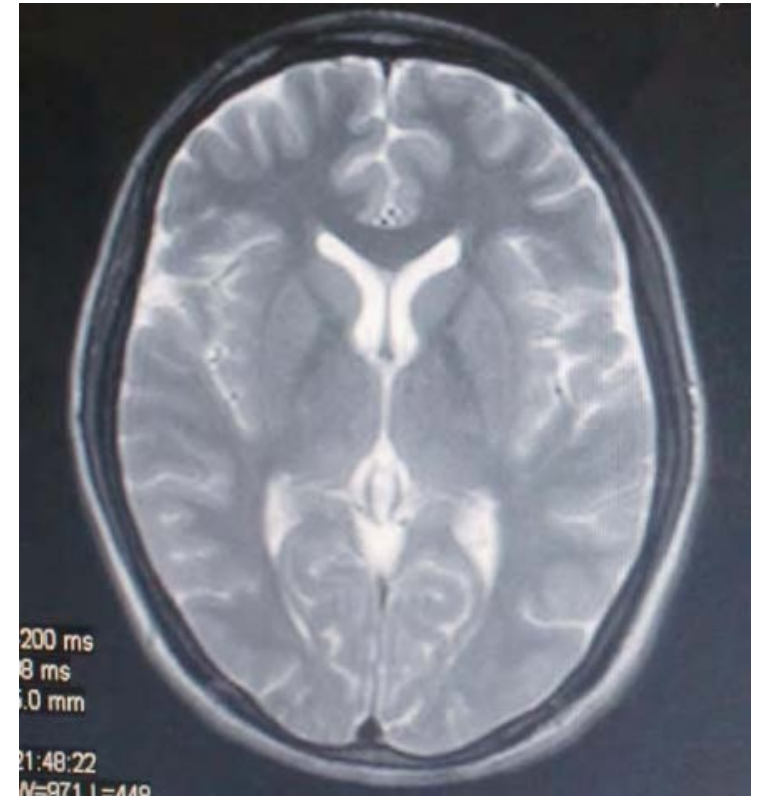

Lumbar puncture(LP) was done which surprisingly revealed opening cerebrospinal fluid(CSF) pressure of $>400 \mathrm{~mm}$ of water along with mildly raised total protein $(0.65 \mathrm{~g} / \mathrm{dl})$, normal cytology results and mildly increased immunoglobulins( $\operatorname{Ig}) \operatorname{IgG}$ $(0.09 \mathrm{mg} / \mathrm{L})$ and $\operatorname{IgA}(27.70 \mathrm{mg} / \mathrm{dl})$.Repeat LP after one week still showed CSF pressure $>400 \mathrm{~mm}$ of water. Her urinary analysis revealed IgA kappa chain $7.72 \mathrm{mg} / \mathrm{dl}$ (0.00-1.85 mg/dl).

Results of blood tests at presentation were remarkable for mild neutrophilic leukocytosis, normal platelets count. And bone marrow biopsy showed hyperplasia with left shift. Neurophysiological examination revealed demyelinating changes in the peripheral nerves.

The diagnosis of POEMS syndrome was made on the basis of the findings and consistent with the established diagnostic criteria (Dispenzieri et al[4]) for the disease. 
We contemplated MR angiogram(MRA) and MR Venogram(MRV) in order to find a cause for highly raised CSF pressure but both MRA and MRV(figure 2) were normal. Ultrasounds of thyroid and breasts revealed breast lobule hyperplasia and thyroid cyst.

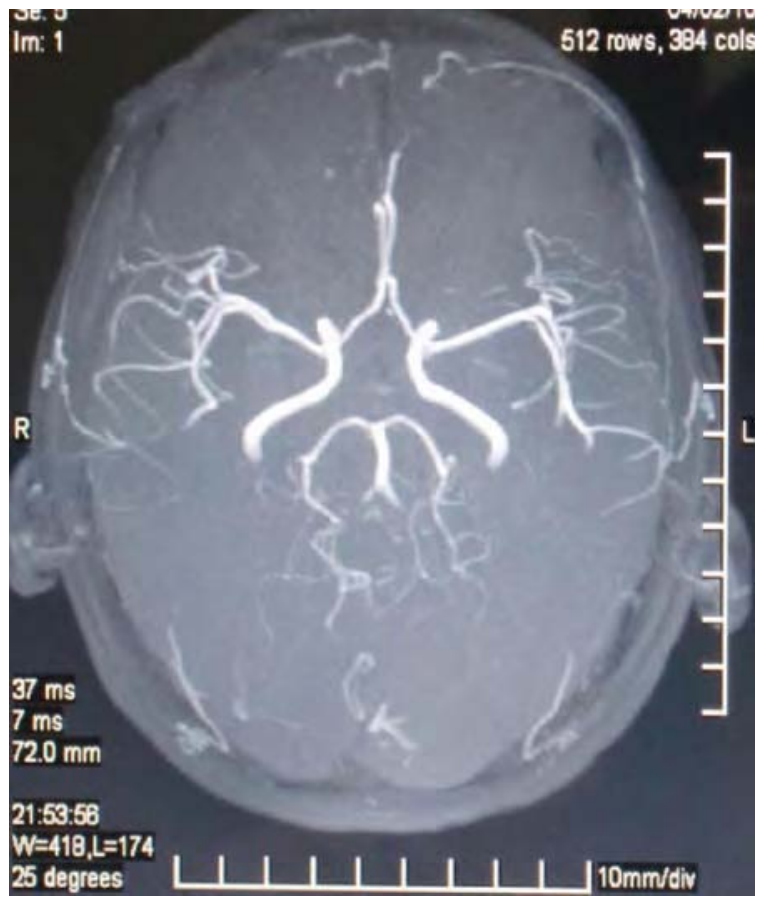

Thoracic and abdominal computed tomography (CT) scans demonstrated organomegaly and ascites as mentioned earlier. No bony lesions were found anywhere in the body.

We considered drug therapy for the POEMS. She was started on steroids and methotrexate. Clinical status improved gradually after aggressive pharmacological management but the patient still could not walk properly. Six weeks later repeat LP revealed opening CSF pressure of $130 \mathrm{~mm}$ of H20. In between this, LP were done for three times which all revealed very high CSF opening pressure $>400 \mathrm{~mm}$ of H20 every time consistent with the initial reading and no other new cytologic, biochemical abnormalities. This time with normalization of the CSF pressure, she also exhibited improvement with her gait. Similarly, USG abdomen showed decreased organ size and extravasted fluids. Overall, she made considerable improvement and was discharged for further management and later follow up in outpatient clinic.

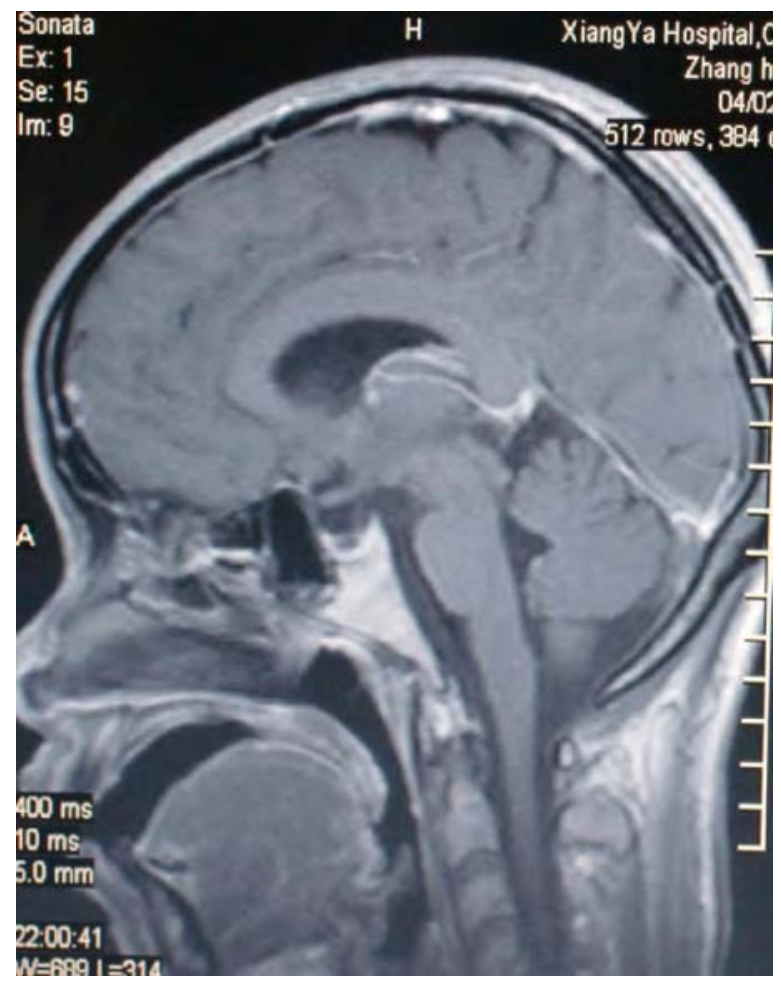

\section{Discussion}

POEMS syndrome is a rare clinical entity. Major diagnostic criteria includes polyneuropathy, monoclonal plasma cell proliferative disorder, sclerotic bone lesions, castleman's disease and elevated vascular endothelial growth factor(VEGF) along with several minor criteria and other associated features [4].The first two major criteria were 
present in our patient though it was the $\operatorname{IgA}$ kappa chain, which is rare, and not the lambda chain isolated in our patient. Serum VEGF level, which is also a major criteria, and serves well to correlate with disease activity was not measured because of lack of laboratory facility. But the presence of the two major criteria and several minor criteria in our case guided us to the correct diagnosis.

The patient in our case presented mainly with symptoms of skin hyperpigmentation followed by a long 'time gap' with hypothyroidism and demyelinating peripheral polyneuropathy. The precedence of a symptom before developing endocrinopathy and other symptoms was in agreement with the findings of the large mayo case series published by gandhi et al [3]. Further investigations revealed hepatosplenomegaly, pleural \& pericardial effusions and ascites indicating extravascular volume overload. These features were too consistent with the findings of a large case series of chinese patients reported by cui et al very recently[8]. The special feature of our case was transient highly raised ICP along with gait disturbance and papilledema at presentation. To our knowledge, transient highly raised opening CSF pressure along with nonspecific gait disturbance had rarely been reported in literature. Very recently, in a small case series of chinese patients reported by Lin et al, the authors reported of raised opening CSF pressure[9].However, they reported mild to moderate increased ICP present inconsistently among patients and did not mention of simultaneous gait disturbance[9]. Papilledema is a common finding in POEMS and had been regularly reported among patients. In our case, we assume that the papilledema was the result of both the disease itself and the highly raised ICP. Various tests including MRA, MRV were carried out to search alternative cause for raised ICP but all were negative.

Arterial and/or venous thrombosis have been occasionally found in POEMS patients culminating into stroke, myocardial infarction, gangrene etc.[3,4]. Several such cases have been reported in literature. One such rare case associated with very high ICP and eventual visual loss and death of the patient with POEMS syndrome had been reported from Thailand [7]. In that case, cerebral venous thrombosis was detected in postmortem autopsy and was considered to cause increased ICP. In our patient, distinct from that reported case, the transient presence of highly raised ICP and gait disturbance at the time of presentation/diagnosis was atypical without any evidence of cerebral venous thrombosis or arterial thrombosis or any alternative cause to explain the finding. We assume that the transient high ICP leading to gait disturbance could be a result of increased vascular permeablity resulting in edema as it is well known that there is extravastation of serous fluid elsewhere in body cavities resulting in ascites, pleural effusion, pericardial effusion etc. in POEMS syndrome[4]. 
This case illustrates the rarity of POEMS syndrome manifestation and presentation with transient highly raised ICP along with nonspecific gait disturbance. Consideration of aggressive medical treatment and surgical intervention for very high ICP is warranted if it remains for too long to prevent visual loss. More detailed case studies and research in future would be required to explain this novel finding and understand the heterogeneity of symptom complex of POEMS syndrome.

\section{References}

1. Bardwick PA., Zvaif er NJ., Gi GN., Newman D., GreenwayDL., Resnick DL. Plasma cell dyscrasia with polyneuropathy, organomegaly, endocrinopathy, $M$ protein, and skin changes: the POEMS syndrome. Report on two cases and a review of the literature. Medicine 1980; 59:311-22.

2. Nakanishi T, Sobue I, Toyokura Y, Nishitani H, Kuroiwa Y, Satoyoshi E, Tsubaki T, Igata A, Ozaki Y. The Crow-Fukase syndrome: a study of 102 cases in Japan. Neurology. 1984 ;34(6):712-20.

3. Gandhi G. , Basu R., Dispezeria A.,Basu A.,Montori V.,Brennan M. Endocrinopathy in
POEMS Syndrome: The Mayo Clinic Experience. Mayo Clin Proc. 2007;82(7):836-842

4. Dispenzieri A.POEMS Syndrome. Review. Blood Reviews. 2007; 21, 285-299

5. Gherardi RK., Be ec L., Soubrier M., et a . Overproduction of proinflammatory cytokines imbalanced by their antagonists in POEMS syndrome. Blood. 1996; 87:1458-1465.

6. Watanabe O., Maruyama I., Arimura K., et a . Overproduction of vascularendothelial growth factor/vascular permeability factor is causative in Crow-Fukase (POEMS) syndrome. Muscle Nerve. $1998 ; 21: 1390-1397$.

7. Witoonpanich R.,Phankhian S., Jootar S., Poonyatha ang A., Surapon Worapongpaiboon S.,Phudhichareonrat S., Chanplakorn N. POEMS Syndrome with Venous Sinus Thrombosis and Visual Failure: A Case Report .J Med Assoc Thai 2005; 88(5): 69

8. Cui R., Huang X., Shi Q., Tian C., Liu J., Pu C.POEMS Syndrome in China. Internal Medicine Journal. 10.1111/j.1445-5994.2009.02172.x8.

9. Lin J, Zhu HL. Clinical Analysis of 22 Cases of POEMS Syndrome. Zhongguo Shi Yan Xue Ye Xue Za Zhi. 2010;18(4):1042-5.

Address of Correspondence: Dr Huan Yang, PhD., Department of Neurology, Xiangya Hospital, Xiangya School of Medicine, Central South University, Changsha, Hunan, 410008, China, E-mail: huany@ymail.com Tel.: +86 731 84327236; fax: +86 73184327401. 